\title{
Experiência de uma disciplina clínica odontológica para pessoas que vivem com HIV/Aids
}

\author{
Thayná Marques Alves*; Amanda Fróes Ribeiro*; Gabriel Felipe Albuquerque Barbosa*; Samuel \\ Trezena**; Edwaldo de Souza Barbosa Júnior***; Carlos Alberto Quintão Rodrigues***; Renata \\ Francine Rodrigues de Oliveira****; Mânia de Quadros Coelho Pinto*****
}

* Estudante, curso de Odontologia, Universidade Estadual de Montes Claros

** Mestrando, Programa de Pós-Graduação em Cuidado Primário em Saúde, Universidade Estadual de Montes Claros

*** Mestre, Professor, curso de Odontologia, Universidade Estadual de Montes Claros

**** Doutora, Professora, curso de Odontologia, Universidade Estadual de Montes Claros

Recebido: 28/06/2021. Aprovado: 08/11/2021.

\begin{abstract}
RESUMO
Considerando-se a importância e necessidade de um ensino odontológico integrado e da propagação do conhecimento sobre a assistência a pacientes que vivem com o HIV/Aids, o curso de graduação em Odontologia da Universidade de Montes Claros (Unimontes) dispõe de uma disciplina específica para o atendimento desses pacientes, a Clínica Integrada IV. O objetivo deste trabalho é relatar a história e a experiência da disciplina Clínica Integrada IV, desde a sua implantação na grade curricular. A disciplina apresenta como objetivo principal propiciar ao acadêmico o conhecimento multidisciplinar e de um planejamento integral do paciente. O atendimento clínico é realizado a partir de um protocolo idealizado pelos professores e constantemente atualizado conforme a literatura. A avaliação odontológica do paciente com HIV/Aids, na Clínica Integrada IV, inclui a investigação da história de infecções oportunistas, de tendência à hemorragia, presença de doenças cardiovasculares, diabetes e hepatites, uso de medicamentos, além da interpretação de exame de sangue recente. É necessário frisar que as normas de biossegurança do atendimento odontológico são preconizadas igualmente para todos os indivíduos, entretanto ainda há uma estigmatização do tratamento odontológico dos indivíduos com o HIV/Aids. Desta forma, uma disciplina com atendimento a pacientes sabidamente soropositivos oportuniza o acadêmico a ter o contato com esse público durante a sua graduação e a desenvolver o conhecimento necessário para se prestar uma assistência específica e com qualidade.
\end{abstract}

Descritores: Assistência Odontológica. HIV. Síndrome de Imunodeficiência Adquirida.

\section{INTRODUÇÃO}

A Síndrome da Imunodeficiência Adquirida (Sida), em inglês Acquired Immunodeficiency
Syndrome (Aids), cujo agente etiológico é o Vírus da Imunodeficiência Humana (HIV), foi considerada pelo Centers for Disease Control 
(CDC), em 1981, como uma doença infecciosa transmissível. Do tipo retrovírus, o HIV é transmitido por via sexual e sanguínea, se reproduzindo no corpo através dos linfócitos TCD 4+, o que leva a uma disfunção imunológica progressiva e crônica e propicia o surgimento das chamadas doenças oportunistas, caracterizando o quadro mais avançado da infecção, que é a Aids ${ }^{1-3}$.

Desde a sua descoberta até a atualidade, a estigmatização gerada em torno do vírus e da doença, que outrora era tida como "sentença de morte", causa impactos negativos na saúde física e mental das pessoas que convivem com o HIV, pois enfrentam, além do medo da doença, preconceitos, discriminação e isolamento social por parte de amigos, familiares e desconhecidos ${ }^{4,5}$.

A epidemia da infecção pelo HIV e Aids tornou-se um problema de saúde pública global que, apesar da queda no número de casos novos em algumas partes do mundo, continua em ascensão. Por exemplo, do Brasil, que no ano de 2019 foram diagnosticados 41.909 novos casos da infecção pelo HIV e 37.308 pessoas com Aids. Deve-se atentar a importância da prevenção dessa doença que depende diretamente de medidas de proteção comportamentais a nível coletivo e individual ${ }^{6}$.

Sua identificação e suas características de transmissibilidade acarretaram mudanças de protocolos de controle de infecção em todas as áreas da saúde, o que levou a um aperfeiçoamento das medidas de biossegurança, inclusive na Odontologia $^{4,7}$.

É necessário frisar que tais normas de biossegurança do atendimento odontológico são preconizadas igualmente para todos os indivíduos, independente do seu status sorológico. ${ }^{8}$ Entretanto, mesmo que a exposição ao HIV durante o manejo é mínima e que, durante a prática, outros microrganismos são mais transmissíveis, como o da hepatite $\mathrm{B}$, ainda há uma estigmatização do tratamento odontológico dos indivíduos com o HIV ou Aids, o que tem acarretado medo e ansiedade em cirurgiões dentistas e feito com que preceitos éticos sejam violados e a assistência negada $^{4,8}$. Essas condutas revelam a falta de conhecimento dos profissionais da odontologia acerca do HIV e da Aids, o que pode comprometer um atendimento clínico seguro e de qualidade ${ }^{2}$.

Dessa forma, percebe-se que há uma grande necessidade da promulgação de conhecimentos que ampliem mais o discernimento dos profissionais cirurgiões dentistas no atendimento aos pacientes que vivem com HIV. ${ }^{9}$ Cabem às clínicas escola o ensino aos futuros profissionais do tratamento aos pacientes com HIV/Aids, desmistificando receios e temores, uma vez que, o contato de acadêmicos com esses indivíduos, durante as clínicas de graduação, são associadas à uma melhor disposição de atendimento durante suas carreiras profissionais ${ }^{10}$. Conforme a Resolução n⿳3 de 21 de junho de $2021^{11}$, a graduação em odontologia, deve propiciar a formação de um profissional que preste uma atenção integral, humana e ética, com base em competências adquiridas durante o curso. A matriz curricular e o projeto pedagógico devem propiciar ao discente o atendimento ao paciente, considerando suas necessidades de saúde, bem como, prepará-los ao atendimento aos indivíduos em instituições de saúde e com necessidades especiais.

Assim, o curso de graduação em Odontologia da Universidade de Montes Claros (Unimontes) dispõe de uma disciplina específica para a assistência desses pacientes, a Clínica Integrada IV, e o objetivo deste trabalho é relatar a história e a experiência vivenciada nessa clínica desde a sua implantação na matriz curricular.

\section{RELATO DE EXPERIÊNCIA}

A Clínica Integrada IV, presente na matriz curricular do curso de Odontologia da Universidade Estadual de Montes Claros (Unimontes), em Montes Claros (MG), iniciou 
suas atividades em agosto de $2001 \mathrm{e}$, desde o seu início, foi realizada por aproximadamente 780 acadêmicos que fornecem assistência à população do município de Montes Claros e de cidades do norte de Minas Gerais. De caráter obrigatória, a disciplina faz parte da matriz curricular do curso da Unimontes, devidamente inserida no Projeto Político Pedagógico, apresentando como objetivo principal propiciar aos acadêmicos, do nono período de graduação, o conhecimento multidisciplinar e de um planejamento integral do paciente.

Com carga horária semestral de 120 horas, a disciplina é dividida em dois momentos diferentes, com o atendimento ao paciente que vive com HIV/Aids (público-alvo do presente relato) e com indivíduos que vivem com deficiências neuropsicomotoras. E nos últimos três anos, a disciplina também começou a propiciar assistência ao paciente oncológico, havendo interface com projetos de extensão da Universidade, que envolvem esses indivíduos. A parte da disciplina, que atende somente pacientes com HIV/Aids, é composta de 33 horas de atividades teóricas, através de aulas com recursos multimídia, grupos de discussões de artigos pertinentes á disciplina, seminários integrados e um trabalho de conclusão de período; e 32 horas práticas em clínicas com atendimento aos pacientes. Sendo critérios de avaliação a aplicação de um prova semestral, a participação efetiva dos docentes nas atividades clínicas e em sala de aula propostas, além da confecção de produtos educativos em saúde, como cartilhas e palestras de educação em saúde.

O atendimento clínico a esse público é realizado a partir de um protocolo idealizado pelos professores e debatido a cada semestre com os acadêmicos, sendo constantemente atualizado conforme a literatura científica. Os pacientes atendidos são encaminhados de centros de referência da região: Hospital Universitário Clemente de Farias (HUCF), Policlínica Alto São
João e do Grupo de Apoio à Prevenção aos Portadores da Aids (Grappa). O manejo desses pacientes leva em conta as etapas do planejamento integral, uma vez que todos têm direito a receber um plano de tratamento, sendo realizados procedimentos de alívio de dor/urgência, avaliação de lesões de tecidos moles, adequação do meio (a partir do reestabelecimento da saúde e conforto bucais), reabilitação, manutenção da saúde bucal e educação em saúde. O plano de tratamento é elaborado pelos acadêmicos, com apoio dos professores da disciplina, mediante análise de riscos, benefícios, limitações e soluções viáveis ao paciente em questão.

A avaliação odontológica do paciente com HIV/Aids, na Clínica Integrada IV, inclui a investigação da história de infecções oportunistas, de tendência à hemorragia, presença de doenças cardiovasculares, diabetes e hepatites, uso de medicamentos, além da interpretação de exame de sangue recente. Essas condutas são importantes para se conhecer o quadro geral do indivíduo, bem como o estágio da doença e o estado imunológico, pois diante dessas informações, o acadêmico consegue estabelecer um planejamento que se adeque à situação do seu paciente, já que modificações no tratamento odontológico podem ser necessárias, com base nas condições gerais de saúde ao longo das sessões de atendimento. Também é estimulado a troca de informações e interconsultas entre os acadêmicos e os médicos que acompanham os pacientes ampliando as habilidades de cuidado interdisciplinar.

A Clínica Integrada funciona como objeto de integração e o eixo de estrutura da disciplina seria o "sistema de atenção à saúde", em que as áreas do saber e do fazer odontológico se unem. A integração de clínicas é benéfica tanto ao paciente, quanto ao acadêmico, que tem sua aprendizagem e habilidade melhoradas. Os acadêmicos se habituam a observar o paciente de forma global, planejando e executando os procedimentos clínicos 
necessários, obtendo controle permanente da evolução e conclusão de tratamentos ${ }^{12}$.

\section{Vivências sentidas e percebidas}

A disciplina de Clínica Integrada IV no seu exercício oferece aos acadêmicos por meio de aulas expositivas, conhecimentos teóricos sobre a história natural, etiopatogenia e manifestações bucais da infecção pelo HIV, os cuidados, as particularidades e orientações quanto aos atendimentos odontológicos aos pacientes, bem como busca também ouvir dos mesmos suas expectativas, receios e disposições para os atendimentos através de grupos de discussões conduzidos por meios de artigos em relação à representatividade social da doença. Os pacientes atendidos, em sua maioria, recebem a assistência odontológica necessária de acordo com o planejamento integral, e contínua em vista da carga horária prática semestral. Alguns pacientes retornam e recebem atenção sequencial com bastante receptividade e cooperação, visando melhoria em sua qualidade de vida, além da ação solidária de todos envolvidos na prestação dos atendimentos devido a relatos de discriminações encontradas em outros serviços públicos e particulares.

Na clínica integrada IV é explicitamente percebida o ganho dos acadêmicos, tanto na execução da prática clínica, a maturidade adquirida, e bem como na segurança ao atendimento do paciente que vive com HIV/Aids, resultante das aulas teóricas prévias ao momento clínico, as discussões individualizadas, realizadas entre as duplas de acadêmicos com seus professores, e pela própria experiência gerada, do atender o paciente que vive com o vírus do HIV. Ainda é observado que o acadêmico se surpreende por identificar que não há fácies típicas ou característica física que especifica o paciente HIV de outras condições de saúde e ele percebe que a condição é encontrada em uma variedade de pessoas de aparência saudável e de diversas faixas etárias convivendo bem com a condição. Também é observado a grande prevalência de omissão do relato da condição de paciente com HIV/Aids na investigação da anamnese, demonstrando que o estigma e a negação da doença ainda são muito predominantes entre os usuários.

É importante ressaltar que a biossegurança utilizada nas práticas clínicas segue as normas instituídas pela Comissão de Biossegurança e Infraestrutura das Clínicas e Laboratórios do Curso de Odontologia da Unimontes ${ }^{13}$, embasadas por análise criteriosa da literatura, leis, portarias e normas técnicas e que durante o momento de pandemia causada pela Covid-19 tiveram que ser modificadas $^{14}$. Além da adoção do uso criterioso de mais equipamentos de proteção individual (EPI), protocolos de desinfecção, dentre outros requisitos para realizar o atendimento na clínica escola, por conta do momento pandêmico, outra medida para minimizar o contágio e propagação do novo coronavírus, optada pela Clínica Integrada IV, foi a redução de dois para um paciente, por dupla de acadêmicos.

\section{O atendimento clínico aos pacientes com HIV/Aids}

O cirurgião-dentista, juntamente com toda sua equipe de trabalho, estão expostos a uma grande variedade de microrganismos presentes no sangue e na saliva do paciente. Diante disso, todo profissional de odontologia deve, por obrigação, adotar normas de prevenção de infecção cruzada, contendo assim, a disseminação de doenças no consultório. Entretanto, apesar das possibilidades de contágio por inúmeros outros microrganismos, nota-se que existe medo e preconceito relacionados à infecção por HIV/Aids, o que reflete no despreparo desses profissionais para oferecer suporte a esses pacientes ${ }^{4,15,16}$.

O despreparo de cirurgiões dentistas em atender pessoas vivendo com HIV/Aids está 
intimamente relacionado ao desconhecimento dos meios de contágio da doença. Trezena et al. $(2020)^{17}$ ao investigarem os fatores que mais contribuem para ocorrência de acidentes durante o atendimento odontológico, obtiveram a falta de treinamento e qualificação como o fator mais relatado. Sabe-se que em acidentes ocupacionais, durante a prática odontológica, havendo contato com sangue e/ou saliva a porcentagem de infecção pelo HIV é de $0,30 \%$, taxa bem inferior quando comparada com os vírus da hepatite (de 06 a $30 \%)^{18}$.

Sabe-se que existe uma dificuldade em identificar pacientes que vivem com HIV, principalmente os assintomáticos, portanto acabam desconhecendo seu status sorológico. É recomendado aos profissionais que sejam tomadas medidas de segurança ao tratarem qualquer paciente, procedendo como se todos tivessem alguma doença infecto contagiosa ${ }^{14,16}$.

Muitos profissionais recusam-se a promover o tratamento destes pacientes ou criam situações que os impossibilitem de realizá-los, ocasionando assim, do ponto de vista ético e legal infrações éticas de acordo com o Código de Ética em Odontologia, uma vez que o paciente está sendo discriminado devido à sua condição sistêmica. Diante disso, faz-se necessário que as instituições de ensino superior ofereçam aos acadêmicos conhecimentos técnicos e uma base alicerçada em fundamentos científicos, para que se sintam seguros durante a realização de procedimentos odontológicos em pacientes que vivem com HIV/Aids ${ }^{4,19}$.

Na mesma universidade em que acontece a Clínica Integrada IV, Costa et al. ${ }^{15}$, em 2009 analisaram a visão dos discentes dos cursos de medicina, educação física e odontologia sobre o atendimento a pacientes HIV/Aids, sendo que 98,52\% dos acadêmicos não deixariam de atender o paciente com essa condição, sugerindo, então, que se sentiam aptos para realizar o atendimento ${ }^{2}$.
Em contrapartida, no estudo de Senna, Guimarães e Pordeus $(2005)^{20}$, realizado com 140 cirurgiões dentistas gerenciados pela Secretaria Municipal de Saúde de Belo Horizonte/MG, houve uma prevalência de $55 \%$ de profissionais que se dispuseram em atender pacientes com HIV/Aids. A menor recepção positiva dos profissionais, a esses pacientes, pode ser associada ao desconhecimento ou até mesmo despreparo dos cirurgiões dentistas durante suas formações.

Contudo, a aceitação ao atendimento odontológico a pacientes com HIV/Aids, pelos estudantes de Odontologia, em estudos internacionais, apresenta resultados variados, com porcentagens inferiores a $29 \%$, dependendo do procedimento a ser realizado ${ }^{21}$. E mesmo em estudos nos quais os acadêmicos apresentavam conhecimento satisfatório sobre a transmissão do HIV/Aids, ainda apresentavam desconforto para o atendimento. Tal informação reforça a importância da contribuição das instituições de ensino superior em fornecer conhecimento detalhado e adequado sobre a temática ${ }^{22,23}$. Com isso, é de suma importância o contato dos profissionais dentistas, durante a graduação, com indivíduos que vivem com HIV/Aids ${ }^{10}$.

No ponto de vista do paciente que vive com HIV/Aids, em uma pesquisa realizada em clínica escola de odontologia em 2019, a análise fenomenológica desvelou que para a pessoa com HIV/Aids o significado de saúde está relacionado ao cotidiano de cuidado, à percepção sobre seu tratamento, à sua experiência com a religião e ao estigma desse adoecimento ${ }^{24}$. Sujeitos com HIV/Aids ainda relatam episódios de discriminação durante o atendimento odontológico mediante suas condições sorológicas, com isso, muita das vezes, o paciente acaba omitindo seu diagnóstico. O principal motivo alegado em $88 \%$ de uma amostra de pacientes para omissão da informação foi o constrangimento devido à sua condição ${ }^{2}$. 
Rossi-Barbosa et al. ${ }^{25}$, buscaram conhecer as expectativas e percepções dos acadêmicos de odontologia da Universidade Estadual de Montes Claros em relação aos atendimentos dos pacientes com HIV/Aids, onde houve uma superestimação do risco de transmissão, mudando seus comportamentos em relação à biossegurança, por medo de contaminação. Necessário frisar que as estigmatizações sociais trazem consigo preconceitos, que podem provocar mudanças no modo em que os usuários dos serviços de saúde, que vivem com o HIV, são acolhidos ou tratados ${ }^{5}$. É essencial reforçar a importância das políticas públicas e o conhecimento dos profissionais de saúde em relação ao HIV, pois a abordagem aberta sobre a temática está relacionada à prevenção de novos casos, a detecção precoce do status sorológico e a redução do preconceito para com as pessoas com HIV/Aids ${ }^{26,27}$.

Nota-se, então, que a prática clínica presente no cotidiano do acadêmico de odontologia, associada à biossegurança, é importante, visto que promove o rompimento de paradigmas $\mathrm{e}$ preconceitos que envolvem o atendimento do paciente HIV/Aids, permitindo, assim, que o paciente receba a intervenção adequada e seja tratado com respeito, compreensão, atenção e solidariedade ${ }^{25}$.

A promoção e orientação em saúde geram mudanças no estilo de vida e contribuem com o bem-estar físico e emocional. Desse modo, o cirurgião-dentista deve se munir de um conhecimento adequado acerca do cuidado de pacientes com HIV/Aids, uma vez que isso resultará no nível de educação, recomendação, motivação e aconselhamento que serão transmitidos ao paciente. Uma atitude positiva frente à assistência a esse público ocasiona em tratamentos e estratégias de prevenção mais exitosos. Além disso, mantêm-se necessárias a realização e veiculação de campanhas regulares e massivas de educação pública, bem como a execução de ações em saúde e de educação continuada, a fim de se melhorar a conscientização sobre a doença, que apresenta uma crescente de casos em alguns países ${ }^{6,28,29}$,

Ademais, é fundamental que se tenha um diálogo aberto com o paciente, para que o mesmo não se sinta discriminado e saiba que as informações coletadas a seu respeito são confidenciais, resguardadas pelos princípios éticos, mas necessárias para fins de diagnóstico, tratamento e pesquisa.

\section{CONSIDERAÇÕES FINAIS}

Uma disciplina com atendimentos a pacientes sabidamente soropositivos para $o$ HIV/Aids oportuniza o acadêmico a ter o contato com esse público durante a sua graduação e a desenvolver o conhecimento necessário para se prestar uma assistência específica e com qualidade. Neste contexto, a Clínica Integrada IV surge com um papel importante, integrando ensino-serviço-comunidade. Apesar de passadas décadas da Aids ter sido considerada como uma doença infecciosa transmissível, ainda existe uma estigmatização das pessoas que vivem com o vírus. Cabe, então, às graduações em Odontologia formar profissionais que estejam cientes da realidade da comunidade a qual se inserem e atendam a população de forma integral e livre de preconceito e discriminação.

O presente relato vislumbra o despertar das instituições de ensino superior na adoção de grades curriculares que possam incluir a temática do paciente que vive com HIV/Aids, formando profissionais sem preconceitos, abertos ao atendimento e conscientes da realidade da assistência a esse público.

\section{AGRADECIMENTOS}

Aos professores Carla Cristina Camilo Araújo, Daniel Neiva Freire, Deícola Coelho Filho, Raquel Conceição Ferreira, Victor Comini Mol e 
Yuri Fonseca Ferreira, que contribuíram com a disciplina Clínica Integrada IV/HIV.

\section{ABSTRACT \\ Experience of a dental clinical discipline for people living with HIV/Aids}

Considering the importance and need for integrated dental education and the dissemination of knowledge about care for patients living with HIV/Aids, the undergraduate course in Dentistry at the University of Montes Claros (Unimontes) has a specific discipline for the care of these patients, the Integrated Clinic IV. The objective of this paper is to report the history and experience of the Integrated Clinic IV discipline, since its implementation in the curriculum. The discipline has as its main objective to provide the academic with multidisciplinary knowledge and comprehensive patient planning. Clinical care is carried out based on a protocol created by the professors and constantly updated according to the literature. The dental evaluation of patients with HIV/Aids, at Clínica Integrada IV, includes an investigation of the history of opportunistic infections, tendency to hemorrhage, presence of cardiovascular diseases, diabetes and hepatitis, use of medications, in addition to the interpretation of a recent blood test. It is necessary to emphasize that the norms of biosafety in dental care are advocate equality for all individuals, however, there is still stigmatization of dental treatment for individuals with HIV/Aids. Thus, a discipline with care for patients known to be HIV positive provides the opportunity for students to have contact with this public during their graduation and to develop the knowledge necessary to provide specific and quality care.

Descriptors: Dental Care. HIV. Acquired Immunodeficiency Syndrome.

\section{REFERÊNCIAS}

1. Paulique NC, da Cruz MCC, Simonato LE, Moreti LCT, Fernandes KCG. Manifestações bucais de pacientes soropositivos para HIV/AIDS. Arch Health Invest. 2017;
6(6):240-4.

2. Costa KS, Griti RC, Brandão FB, Maia PRM, Steinhauser HC, Gritti GC. Aspectos Éticos Relacionados ao Atendimento Odontológico de Pacientes HIV Positivo. Rev Bras Odontol Leg RBOL. 2020; 7(2):2-10.

3. Gir E, Prado MA, Canini Silvia RMS, Hayashida M. O Impacto da AIDS na Prática da Enfermagem: Um Problema de Saúde Pública. DST - J Bras Doenças Sex Transm. 2005; 17(1): 39-43.

4. Discacciati JAC, Vilaça EL. Atendimento odontológico ao portador do HIV: medo, preconceito e ética profissional. Rev Panam Salud Pública. 2001; 9(4):234-9.

5. Carvalho SM, Paes GO. A influência da estigmatização social em pessoas vivendo com HIV/AIDS. Cad Saúde Colet. 2011; 19(2): 157-63.

6. Brasil, Ministério da Saúde. Boletim Epidemiológico: HIV/AIDS. Secretária de Vigilância em Saúde/Ministério da Saúde. Dezembro - 2020. [Acesso em 23 jun. 2021] Disponível em: http://www.aids.gov.br/ptbr/pub/2020/boletim-epidemiologico-hivaids $\underline{-2020 .}$.

7. Rodrigues MP, Sobrinho MD, Silva EM. Os cirurgiões-dentistas e as representações sociais da Aids. Ciênc Saúde Colet. 2005; 10(2): 463-72.

8. Reznik DA, Croser D, Kadrianto TH, Lavanya R. Ethics, research and HIV: lessons learned- a workshop report. Oral Diseases. 2016; 22 (Suppl. 1):193-8.

9. Parola GB, Zihlmann KF. A saúde bucal na perspectiva das pessoas vivendo com HIV/Aids: subsídios para a educação permanente de cirurgiões-dentistas. Interface (Botucatu). 2019; 23:e180441.

10. Nascimento CF, de Souza GS, Vitor LKS, Varejão LC, Azulay MS. Desmistificando o atendimento odontológico para paciente 
soropositivo: Revisão de Literatura. Braz J Develop. 2020; 6(11): 91634-52.

11. Brasil. Ministério da Educação. Conselho Nacional de Educação. Resolução n ${ }^{\circ} 03$, de 21 de junho de 2021. Institui as Diretrizes Curriculares Nacionais do curso de graduação em Odontologia e dá outras providências. Diário Oficial da União. Brasília, 2021. [Acesso em 12 out. 2021]. Disponível em: https://www.in.gov.br/web/ dou/-/resolucao-n-3-de-21-de-junho-de-20 21-327321299.

12. Milani PAP. Avaliação e produtividade da disciplina de clínica integrada no curso de odontologia da Universidade de Tuiuti do Paraná. Dissertação, Pós-graduação em Odontologia. São Paulo. 2003. [Acesso em 23 jun. 2021]. Disponível em: https://teses.usp.br/teses/disponiveis/23/2313 8/tde-09092003-084212/pt-br.php.

13. Universidade Estadual de Montes Claros. Gabinete do Reitor. Portaria $\mathrm{n}^{\circ} 094$ Reitor/2010. Constitui a Comissão de Biossegurança e Infra-estrutura das Clínicas e Laboratórios do Curso de Odontologia, vinculado ao Centro de Ciências Biológicas e da Saúde (CCBS). Montes Claros: Unimontes, 2010. [Acesso em 24 jun. 2021]. Disponível em: https://unimontes.br/wpcontent/uploads/2019/05/portarias/2010/port aria_reitor094.pdf.

14. Barbosa GFA, Ribeiro AF, Nobre MCO, Lima RFR, Oliveira CC, Bonfim MLC, Ferreira SM, Pinto MQC. A biossegurança em tempos de covid-19 no Curso de Odontologia da Unimontes: relato de experiência. Unimontes Científica. 2020; 22(2):1-12.

15. Costa SM, Dias OV, Canela JR, Drumond JGF, Santa-Rosa TTA. Visão de Discentes sobre Atendimento a pacientes HIV/AIDS. Arq Odontol. 2009; 45(1):10-5.
16. Jorge AOC. Princípios de Biossegurança em Odontologia. Rev Biociênc. 2002; 8(1):7-17.

17. Trezena S, Farias LPM, Barbosa GFA, Costa SM, Barbosa-Júnior ES, Pinto MQC. Práticas em biossegurança frente aos acidentes ocupacionais entre profissionais da odontologia. Arq Odontol. 2020; 56:e07.

18. Ministério da Saúde. Secretaria de Vigilância em Saúde. Departamento de Vigilância em Saúde Ambiental e Saúde do Trabalhador. Exposição a Materiais Biológicos - Saúde do Trabalhador: Protocolos de Complexidade Diferenciada. Série A Normas e Manuais Técnicos. Brasília - DF, 2011. [Acesso em 24 jun. 2021]. Disponível em: http://www1.saude.rs.gov.br/dados/1332967 170825PROTOCOLO $\% 20$ EXPOSICAO $\% 2$ 0A\%20MATERIAL\%20BIOLOGICO.pdf

19. Conselho Federal de Odontologia (CFO). Código de Ética em Odontologia: resolução no. 42, de 20 de maio de 2003. Rio de Janeiro; CFO; 2006. [Acesso em 24 jun. 2021]. Disponível em: http://sinog.com.br/downl oads/\%7BFAC08150-034D-4F69-A683-

3FF6121A9FCD\%7D_codigo_etica.pdf

20. Senna MIB, Guimarães MDC, Pordeus IA. Atendimento odontológico de portadores de HIV/AIDS: fatores associados à disposição de cirurgiões-dentistas do Sistema Único de Saúde de Belo Horizonte, Minas Gerais, Brasil. Cad Saúde Pública. 2005; 21(1): 21755.

21. Katwal D, Rimal J, Prajapati K. Knowledge, attitude and practice of dental students regarding treatment of patients with HIV or AIDS in Nepal. Kathmandu Univ Med J (KUMJ). 2019; 17(67):190-4.

22. Grover N, Prakash A, Singh S, Singh N, Singh P, Nazeer J. Attitude and knowledge of dental students of National Capital Region regarding HIV and AIDS. J Oral Maxillofac Pathol. 2014;18(1):9-13. 
23. Jin CX, Meng YC, Du WZ, Pei DD, Li A. Knowledge of infection prevention and attitudes towards HIV/AIDS Among Chinese Dental Bachelor Interns: an appeal for educational intervention. Oral Health Prev Dent. 2020;18(1):485-92.

24. Pinto MQC, Martins TCR, Mendonça JMG, Costa SM, Sampaio CA. Saúde na perspectiva de pessoas com HIV/aids: um olhar fenomenológico. Temas Saúde. 2019; 19(3): 484-96.

25. Rossi-Barbosa LAR, Ferreira RC, Sampaio CA, Guimarães PN. "Ele é igual aos outros pacientes": percepções dos acadêmicos de Odontologia na clínica de HIV/Aids. Interface (Botucatu). 2014; 18(50):585-96.

26. Dong X, Yang J, Peng L, Pang M, Zhang J, Zhang Z, et al. HIV-related stigma and discrimination amongst healthcare providers in Guangzhou, China. BMC Public Health. 2018; 18(1): 738, 2018.
27. Getter A, Herron AR, Sutton MY. HIVRelated Stigma by Healthcare Providers in the United States: A Systematic Review. AIDS Patient Care STDS. 2018; 32(10): 418424.

28. Oliveira JK,Vieira LE, Limeira MS, Araújo MG, Oliveira DH, Chacon LD, et al. Práticas educativas em saúde bucal direcionada aos usuários do restaurante popular da cidade de Patos-PB. Rev Bras Ext Univ. 2017;8(2):6774.

29. Ali A, Ali NS, Nasir U, Aadil M, Waqas N, Zil-E-Ali A, et al. comparison of knowledge and attitudes of medical and dental students towards HIV/AIDS in Pakistan. Cureus. 2018;10(4):e2426.

\section{Correspondência para:}

Samuel Trezena

e-mail: samueltrezena@gmail.com

Rua Lagoa Escura, 406 Carmelo

39402-704 Montes Claros/MG 